\title{
Collapsar Disks and Winds
}

\author{
A. I. MacFadyen \\ Caltech, MC 130-33, Pasadena, CA 91125, USA
}

\begin{abstract}
.
Winds blown from collapsar accretion disks may produce observable stellar explosions independent of any GRB-(and afterglow)-producing jets which may be simultaneously produced. The production of winds is controlled by the accretion disk physics, in particular, the nature of disk cooling via neutrino emission and photodisintegration of heavy nuclei. These temperature-dependent processes depend on the stellar angular momentum via the depth of the gravitational potential at the Kepler radius where the disk forms. Wind-driven stellar explosions which do not make a GRB (or only a faint one) may occur and constitute a new class of supernova explosion. SN1998bw and 1997ef may be examples. A key feature of collapsar winds is that they are capable of producing the radioactive ${ }^{56} \mathrm{Ni}$ necessary to power a supernova light curve. It is possible to make a GRB in a star without significant production of ${ }^{56} \mathrm{Ni}$. Such a star would not make an observable supernova and no such component would be expected in the light curve of the optical afterglow.
\end{abstract}

\section{INTRODUCTION}

Simulations of the collapse of massive rotating stars indicate the viability of rotating stellar collapse (collapsars) as the central engine for the long duration gamma ray bursts[1][2].

Collapsars form dense accretion disks $\left(\rho \gtrsim 10^{9} \mathrm{gm}\right.$ $\mathrm{cm}^{-3}$ ) which are extremely optically thick to photons $\left(\tau_{\gamma} \sim 10^{19}\right)$. As the stellar gas spirals through the disk, photons are trapped and accrete with the gas. This is in distinction from "thin" accretion disks in which photons are assumed to escape to infinity carrying away the locally dissipated energy. Since photons are trapped, viscous dissipation of orbital energy increases the disk entropy, pressure gradients are important for the force balance and the disk is "thick." Such non-radiating accretion flows are capable of ejecting gas away from the black hole [3, e.g.]. Accretion in these disks is inefficient with significant fractions of the gas supplied at large radii being ejected from the system.

An important feature of collapsar disks, is the realization at sufficiently high accretion rates $\left(\dot{M} \sim 0.1 M_{\odot} s^{-1}\right)$ of temperatures $\left(T \sim 10^{10} \mathrm{~K}\right)$ and densities $\left(\rho \sim 10^{9} \mathrm{gm}\right.$ $\mathrm{cm}^{-3}$ ) at which the loss of thermal energy to neutrino emission and photodisintegration of heavy nuclei allows for accretion with a range of efficiency.

\section{WINDS}

MacFadyen \& Woosley 1999 showed that collapsar disks eject comparable amounts of material in a wind as is accreted by the central black hole. The fraction of accreted gas depends on the efficiency of neutrino cooling at removing entropy from the accreting gas. The remainder is ejected from the black hole as an outflowing wind. Recent semi-analytic work [4] has mapped the parameter space of inefficient neutrino-cooled accretion in agreement with detailed calculations of [1] for limited parameters.

Of particular interest in the case of collapsars is the chemical composition of the wind. Collapsar disks are hot enough to completely photodisintegrate heavy nuclei to free nucleons (neutrons and protons). Recent simulations [5] and [1] show expulsion of free nucleon gas in the wind. This is important for two reasons: 1) energetics: free nucleons combining to iron group nuclei (e.g. Nickel-56) release $8 \mathrm{MeV} /$ nucleon or $1.5 \times 10^{52} \mathrm{erg}$ per solar mass of recombined material. 2) observability: this ejected material provides a long term energy supply to the explosion (through radioactive decay of ${ }^{56} \mathrm{Ni}$ ) enabling the gas to shine on time scales of months.

\section{LIGHT CURVE}

Models of the light curve of the energetic and peculiar Type Ibc supernova SN1998bw require large quan- 
tities of ${ }^{56} \mathrm{Ni}\left(\mathrm{M}\left({ }^{56} \mathrm{Ni}\right) \sim 0.5 M_{\odot}\right)$ [6][7]. Conventional models require large explosion energies to produce sufficient nickel and fit the light curve. In addition abnormally high expansion velocities were inferred from the unusual spectrum indicating a large explosion energy $\left(\sim 10^{52} \mathrm{erg}\right)$. Several groups have also interpreted deviations from power law decay of GRB optical transients as supernovae light curve components and have matched them with appropriately shifted 1998 bw light curves [8, e.g.].

Since supernovae are invoked to interpret these observations it is important to note that a stellar explosion (e.g., jets piercing a star) is not necessarily a supernova. Supernovae, as an observable phenomenon, require a persistent source of energy input to power a light curve for long times (weeks to months). It is necessary to make ${ }^{56} \mathrm{Ni}$ in the explosion so that radioactive decay (to Cobalt to Iron) injects energy into the gas so that it can shine. Lacking a persistent source of energy input, a stellar explosion would be unobservable via electromagnetic radiation. Explosion energy released in the optically thick star would simply be converted to expansion kinetic energy with little or no light emitted.

In conventional core collapse supernovae some nickel is thought to be produced via explosive nuclear burning behind the explosion shock. However, current models for these "delayed" supernova explosions have trouble producing the $10^{51}$ erg for a normal supernova (in fact, some current models fail to get any explosion at all!) and are unlikely to be capable of producing the higher energies required for $1998 \mathrm{bw}$.

\section{ANGULAR MOMENTUM}

As we have seen, neutrino cooling and photodisintegration of heavy nuclei are crucial for allowing gas to accrete efficiently. The neutrino cooling depends sensitively on temperature (e.g., $Q_{v}^{-} \propto T^{6}$ for neutrino losses due to pair capture on free nucleons) and therefore on the radius where the disk forms. This radius is, in turn, dependent on the angular momentum of the accreting gas with the disk first forming at the Kepler radius $R_{\text {kep }} \equiv j^{2} / G M=2.5 \times 10^{7} j_{17}^{2} M_{3}^{-1} \mathrm{~cm}$, where $j_{17}$ is the specific angular momentum of the accreting gas in units of $10^{17} \mathrm{~cm}^{2} \mathrm{~s}^{-1}$ and $M_{3}$ is the mass of the central black hole in units of three solar masses. The virial temperature for gas falling to its Kepler radius $T_{\text {vir }}=G M m_{p} / 3 k_{B} R_{\text {kep }}=3.3 \times 10^{10} M_{3}^{2} j_{17}^{-2} \mathrm{~K}$, where $m_{p}$ is the proton mass and $k_{B}$ is the Boltzmann constant. In terms of gravitational radii, $R_{G} \equiv G M / c^{2}$, this temperature is $T_{v i r}=m_{p} c^{2} / 3 k_{B} r=1.8 \times 10^{12} r^{-1} \mathrm{~K}$ (assuming a Newtonian potential), where $r \equiv R / R_{G}$. We see that gas with $j_{17} \approx 1$ is heated to above $10^{10} \mathrm{~K}$ so that it is fully photodisintegrated to free neutrons and protons from it's original composition of Silicon, Oxygen and Helium. This means that capture of electron-positron pairs onto the free neutrons and protons serves as an efficient neutrino emission process which cools the gas and helps it to accrete efficiently. Gas with $j_{17} \gtrsim 2.6$, however, heats to less then $5 \times 10^{9} \mathrm{~K}$. At these lower temperatures the heavy nuclei fail to photodisintegrate and pair capture neutrino cooling is suppressed. This gas is therefor poorly cooled and subject to being driven from the disk.

It is worth noting that gas with $1 \lesssim j_{17} \lesssim 2.6$ is partially photodisintegrated. Photodisintegration acts as a loss for thermal energy for the gas and thus is effectively a cooling process, robbing about $10^{19}$ erg of thermal energy from every gram of photodisintegrated nuclei. This process helps the gas to accrete and provides free nucleons which enhance the neutrino cooling.

The above discussion assumed $M_{3}=1$ though the scaling with $M_{3}$ is apparent.

\section{THE AFTERBURNER}

Interestingly, energy lost to photodisintegration becomes available again if the gas is ejected from the disk and begins to reassemble.

Effectively, gravitational energy is temporarily stored in the freeing of nucleons from the heavy nuclei. These nucleons are volatile in the sense that the have a huge nuclear energy source if they manage to escape the energetic photons trapped in the (optically thick) accretion disk. Accretion physics may provide the nucleons with opportunity to escape the disk's nuclei-disintegrating photon bath. Once free they can quickly recover nuclear binding energy by reassembling into iron group elements. This process can be explosive since the nuclei may recombine in seconds compared to millions of years it took them to assemble (burn) the first time around during the slower pre-explosion nuclear burning stages. In fact the reassembly, plus the kinetic luminosity of the disk wind, may power extremely energetic explosions. SN1998bw may be an example.

\section{GRB WITH SUPERNOVA}

The collapsar model relies on rapid accretion into the central black hole to power relativistic jets which pierce the star and make a GRB and afterglow via internal and external shocks. It is notable that for an interesting range of angular momentum the accretion of the star simultaneously feeds the black hole rapidly and powers a wind [1]. 


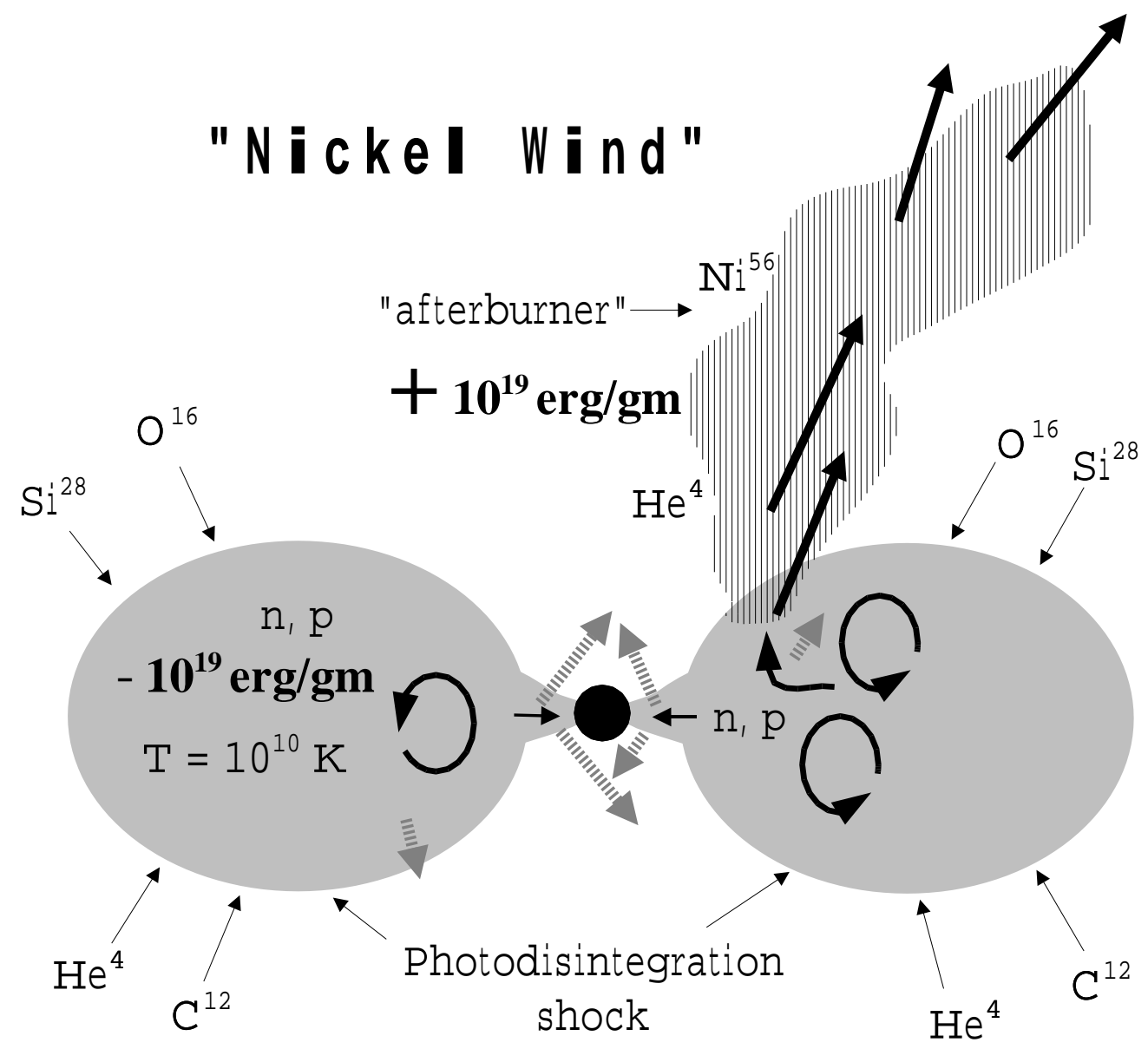

FIGURE 1. Cross section of a collapsar accretion disk feeding a stellar mass black hole (center). The disk is embedded in a collapsing star that is falling onto the disk at rates above $0.1 \mathrm{M}_{\odot} \mathrm{s}^{-1}$. A wind (the striped region in the upper right) is blown from the collapsar disk at speeds of up to $\sim 40,000 \mathrm{~km} \mathrm{~s}^{-1}$. The wind is composed of free neutrons and protons which can recombine to iron group elements injecting $1.5 \times 10^{51}$ erg per $0.1 \mathrm{M}_{\odot}$ of reassembled nucleons. ${ }^{56} \mathrm{Ni}$ in the wind can power a long term "supernova" light curve via radioactive decay of nickel and cobalt. The black solid arrows indicate the velocity of the gas flow while the thick dashed lines represent neutrino emission. The wind is shown only in the upper right quadrant for clarity but is in reality present in all four quadrants. 
There are three interesting regimes determined by the angular momentum present in the collapsing star:

The following values of angular momentum correspond to important transition radii in the accretion flow:

$j_{i s c o}$ angular momentum of the innermost stable circular orbit. This is the minimum angular momentum needed to form a disk around a black hole.

$j_{v}$ angular momentum of gas that falls deep enough in the gravitational potential to photodisintegrate the heavy nuclei to free nucleons activating pair capture neutrino emission as an efficient coolant.

$j_{\gamma}$ angular momentum of gas that falls deep enough to cool partially. Some gas accretes and some is expelled in a wind. The relative amount depends on the exact value of $j$

1. $j_{i s c o}<j<j_{v}$ - efficient neutrino cooling allows rapid accretion into black hole with plenty of power potentially going into jets with little or no outflows expected. This kind of star would not be expected to produce a bright supernova since little or no ${ }^{56} \mathrm{Ni}$ is expected to be present in the exploding star. A possible caveat is that there is some nickel production via explosion burning in the lateral jet shock but the temperature is low in this region and not much mass is involved.

2. $j_{v}<j<j_{\gamma}$-some gas accretes and some is ejected in a wind rates can be comparable depending on $\mathrm{j}$. This can make both a GRB and a "supernova"

3. $j>j_{\gamma}$ Gas doesn't cool efficiently so doesn't feed the hole rapidly. Not good for making an accretion powered GRB. Outflows may results with some recombination nickel possible if some gas is heated above $5 \times 10^{9}$ $\mathrm{K}$ by a combination of virialization and viscous dissipation. Of interest here is explosive burning of centrifugally supported oxygen.

A less interesting regime is $j<j_{l s o}$ for which the gas falls directly to the innermost stable circular orbit without forming an accretion disk.

Note that electromagnetic extraction of black hole spin energy is a possible source of jet energy even for "slowly" accreting black holes. Convective motions may even be favorable for building up large magnetic fields needed to extract the hole spin energy.

\section{VISCOSITY}

The above scenario assumes significant viscosity in the disk gas corresponding to a Shakura-Sunyaev alpha viscosity parameter $\gtrsim 0.1$. The temperature and density of the disk wind and hence the nucleosynthesis depend on the disk viscosity. Observations of of the supernova powered by a collapsar wind may help to constrain the viscosity of the collapsar accretion disk.

\section{WORK IN PROGRESS}

Detailed hydrodynamical simulations of the disks and winds described here using the FLASH code are currently underway.

\section{REFERENCES}

1. MacFadyen, A. I., and Woosley, S. E., ApJ, 524, 262-289 (1999).

2. MacFadyen, A. I., Woosley, S. E., and Heger, A., ApJ, 550, 410-425 (2001).

3. Hawley, J. F., Balbus, S. A., and Stone, J. M., ApJ, 554, L49-L52 (2001).

4. Narayan, R., Piran, T., and Kumar, P., ApJ, 557, 949-957 (2001).

5. MacFadyen, A. I., and Woosley, S. E., ApJ, in preparation (2002).

6. Iwamoto, K., Mazzali, P. A., Nomoto, K., Umeda, H., Nakamura, T., Patat, F., Danziger, I. J., Young, T. R., Suzuki, T., Shigeyama, T., Augusteijn, T., Doublier, V., Gonzalez, J.-F., Boehnhardt, H., Brewer, J., Hainaut, O. R., Lidman, C., Leibundgut, B., Cappellaro, E., Turatto, M., Galama, T. J., Vreeswijk, P. M., Kouveliotou, C., van Paradijs, J., Pian, E., Palazzi, E., and Frontera, F., Nature, 395, 672-674 (1998).

7. Woosley, S. E., Eastman, R. G., and Schmidt, B. P., ApJ, 516, 788-796 (1999).

8. Bloom, J. S., Kulkarni, S. R., Djorgovski, S. G., Eichelberger, A. C., Cote, P., Blakeslee, J. P., Odewahn, S. C., Harrison, F. A., Frail, D. A., Filippenko, A. V., Leonard, D. C., Riess, A. G., Spinrad, H., Stern, D., Bunker, A., Dey, A., Grossan, B., Perlmutter, S., Knop, R. A., Hook, I. M., and Feroci, M., Nature, 401, 453-456 (1999). 